\title{
ZOONOTIC AND HISTO-PATHOLOGICAL ASPECTS OF VARIOUS TOXOPLASMA GONDII STRAINS IN FEMALE RATS
}

\author{
El Fadaly H. A.* Soror A. H. **, Barakat A. M. A. *, Abd El-Razik K. A**
}

*Zoonotic Department, National Research Center, Egypt, ** Animal Reproduction Department, National Research Center, Egypt

Corresponding author: khaled707@ hotmail.com, khaledemara707@yahoo.com

\begin{abstract}
Background: Toxoplasma gondii is one of the most opportunistic zoonotic protozoan, typifying three virulent types; I, II, and III; with possible characteristic pathological and serological aspects, showing zoonotic and reproductive impact. The present investigation dealt with the concept, and focused on studying the susceptibility of female rats to cerebral and uterine toxoplasmosis, with reference to diversity of $T$. gondii virulent types through histopathological and serological assays.

Materials and Methods: A total of thirty pathogen free Sprague-Dawley female rats were used for the study. They were divided into two main groups (acute and chronic) groups: Both were further subdivided into three additional subgroups in correspondence with the three T. gondii types; I, II and III (local isolates) strains. ELISA IgM / IgG as well as pathological investigations of visceral organs; brain and muscles were done prior to 7 days post Inoculation. (DPI) and $35 \mathrm{DPI}$, represent the acute and chronic stages respectively.

Results: The results revealed that type I possess, a higher percentage for inducing cerebral and uterine toxoplasmosis in addition to higher elevation of both parasite load and immunoglobulin titers; while cystogenic types II and III showed lower values.

Conclusion: It could be concluded that; successful recognition of cerebral and uterine toxoplasmosis were sequential to the three $T$. gondii virulent types, and reflects the possible match of pathological and serological alters with humans at similar circumstance.
\end{abstract}

Key words; T. gondii strains, cerebral and uterine toxoplasmosis, ELISA, Pathology

\section{Introduction}

Toxoplasmosis is an acute or chronic opportunistic zoonosis, which exceeds public health hazards and particularly sequential to severe cerebral and congenital human toxoplasmosis. Toxoplasma gondii is an obligate intracellular protozoan, whose acute tachyzoite stage is responsible for the materno-fetal and cerebral diffusion. While the chronic bradyzoite stage (tissue cyst) remapablein viable for the rest of the host survival, can revert acute tachyzoites (latent infection) consequence to unaccepted stressors. Both, immunological (Jones et al., 2001) and corticosteroid therapy (Elfadaly, 2007) in addition to sex steroids hormonal shift (Elfadaly et al., 2012) were documented as the major factors capable of leading to opportunist latent toxoplasmic encephalitis.

Cerebral or congenital human toxoplasmosis usually results into latent opportunity, and posses a serious threat during pregnancy and in immunesuppressed personnel, resulting into enhanced risks of fetal transmission in about 30\% and may develop predominant mental retardation, loss of vision, fetal death, abortion, and stillbirths, and neurological sequel of congenital malformations, in addition to ocular disorders (Tenter et al., 2000; Louis et al., 2007). Also toxoplasmic encephalitis was considered the third most deadliest cause of immune-suppressed patients infections (Kovari et al., 2010), while cystogenic type II was set as the predominant latent brain cyst forming strain (75\%) in congenital and cerebral human toxoplasmosis (Boothroyd , 2009; Zhou et al., 2004; Hassanain et al.,2013).

Toxoplasmosis affects a wide range of different animal species including small ruminants, equines and rabbits, and causes serious economic losses especially within the sheep industry across the globe, especially at the time of lambing, fetal re-asorption, abortion, fetal mummification, stillbirth, or birth depletion (Barakat et al.,2005; Abd El-Razik et al., 2011; Shaapan et al., 2012).

Most $T$. gondii strains are virulent, whereas the three types I, II and III exhibit higher virulent profile with clinical, epidemiological and zoonotic variability; Type I strain being uniformly acute lethal poorly-cyst forming in outbreed mice (LD100_1) and leads to death of mice in less than 10 days after inoculation of < 10 tachyzoites; in contrast, types II and III are considerably less virulent cyst-forming strains with neurological symptoms Barakat, et al., (2001). Given such biological diversity we could expect dissimilar pathological courses corresponding to acute and chronic infection with different types. Since the course of toxoplasmosis is not controlled in human, it was advanced to perform animal model so as to confirm different strains bio-hazard quotient. Diagnosis of $T$. gondii has traditionally been based on the characteristic histo-pathological changes (Desouky et al., 2005).

Thus, the objective of the present study was to evaluate the pathological and serological possibilities of cerebral anduterine toxoplasmosis using acute and chronic experimentally infected female rats; to reflect to what extent the possible human-neurological and reproductive bio-hazards sequence is to different types. Also, the results copy parallel histo-pathological and serological findings when compared with cerebral and congenital human toxoplasmosis.

\section{Materials and methods \\ Toxoplasma gondii strains}

In this study, three strains RH, ME-49 and Prugniaud (PRU) strains were secured from Zoonotic Diseases Department, National Research Center, Egypt; as representing types I, II respectively, and type III (local isolates) with the following lethal doses: LD100 1, LD50 10², and LD50 $10^{3}$ respectively in outbreed mice. The RH strain tachyzoites were maintained through successive intra-peritoneal tachyzoites-tachyzoites passages in mice every 3 days, the obtained tachyzoites were used for intra-peritoneal infection after counting and dilution was done as required $\left(10^{5}-10^{6}\right.$ factor dilution).

The ME-49 and PRU strains (cystogenic strains) were maintained by oral inoculation of brain cysts in mice every 3 months. Under sterile conditions, brain from infected mice were homogenized in 20-ml potter's tube, and brain cysts were counted and diluted as required (5-20 factor dilution), ready for oral or intra-peritoneal injection for continuous passage in mice (Carneiro et al., 2009). 
http://dx.doi.org/10.4314/ajid.v9i2.3

Animals

30 pathogen free Sprague-Dawley female rats obtained from the Laboratory Animals House, National Research Center, Egypt; were used in acute and chronic experimental design.

Table 1: Experimental design

\begin{tabular}{lllllll}
\hline Types & Strains & Infective stage & $\begin{array}{l}\text { Acute } \\
\text { (7DPI) }\end{array}$ & $\begin{array}{l}\text { Chronic } \\
\text { (35DPI) }\end{array}$ & Control & Total \\
\hline Type I & RH & Mice peritoneal tachyzoites & 3 & 5 & 2 & 10 \\
Type II & ME-49 & Brain tissue cysts (bradyzoites) & 3 & 5 & 2 & 10 \\
Type III & Prugniaud (PRU) & Brain tissue cysts (bradyzoites) & 3 & 5 & 2 & 10 \\
Total & & & $\mathbf{9}$ & $\mathbf{1 5}$ & $\mathbf{6}$ & $\mathbf{3 0}$ \\
\hline
\end{tabular}

The animals were housed in standard environmental conditions of temperature $\left(24{ }^{\circ} \mathrm{C}\right)$ and relative humidity $(50 \%)$ with a $12: 12$ light/dark cycle. with free access to standard commercial diet and water. Experiments were performed in accordance with the Guide for the Care and Use of Laboratory Animals and Ethical Approval of animal rights.

\section{Blood and tissue samples}

A total of 30 samples of both blood and tissue were collected from acute ( $n=9)$, chronic $(n=15)$ and control ( $n=6)$ groups, corresponding to the three types; I, II \& III. Tissue samples were collected from all acute and chronic female rats from; L (liver), K (kidney), P (pancreas), U (uterus), B (brain) and $\mathrm{M}$ (skeletal muscles).

\section{IgM /IgG ELISA antibody titers}

Sera were immediately separated by centrifugation of rat blood samples at $3000 \mathrm{rpm}$ for $10 \mathrm{~min}$, sera were kept at $-20{ }^{\circ} \mathrm{C}$ until examined by ELISA using IgM /IgG ELISA diagnostic kits (VIRO, Germany), the procedure was done according the methods described by (Lind et al. ,1997)

\section{Parasite load estimation}

Emulation of different experimented tissues were prepared by homogenization with an equal volume of Phosphate Buffer Saline (PBS) pH 7.4 and passed through 16-gauge needle ten times by means of a syringe to release tissue cysts (Dubey and Beattie, 1988). One drop of the homogenate was spread on a slide and the parasite was microscopically counted in average of every $10 \mathrm{ml}$ using haemocytometer. The average parasite load (APL/10 ml) was practically evaluated through counting the total number of cyst-free bradyzoites plus in cysts bradyzoites /gm ( Hassanain, et al., 2012).

\section{Histo-pathological methods}

Tissue samples were taken from brain, lungs, liver, kidneys, heart and uterus of the three groups of rats treated with different strains of Toxoplasma gondii (RH strain, ME49 strain and local isolates) uterus Samples were fixed in 10\% neutral buffered formalin. Washed, dehydrated and embedded in paraffin wax. The tissues were sectioned at 4-5 thickness and stained with haematoxylin and eosin (H and E) as routine work for histopathological examination according to Bancroft et al., (1996). Periodic acid-Schiff reaction "PAS" (Drury and Wallington 1980) and Toluidine blue stains (Drury et al., 1976) were used were for detection of T. gondii within the tissue.

\section{Statistical analysis}

Data were statistically analyzed using the MSTAT and STATISTICA (6.0) computer programs. The Average and Standard Deviation among the different parameters were determined as well (Godard et al., 1990). Only differences with a probability of error of less than 0.05 were considered significant.

Ethical Approval: The work was approved ethically by the Medical Research Ethics Committee-National Research Centre, Al Tahrir St. Dokki, Giza, Egypt under registration number 1-2 /0-2-1.2012.

\section{Results}

Serological results

Table-2:_Average parasite load values of both acute and chronic stages in different organs, corresponding to different strain types and serological titers.

\begin{tabular}{|c|c|c|c|c|c|c|c|c|c|c|c|c|c|c|}
\hline & \multicolumn{7}{|c|}{ Average values of acute stage at (7 DPI) } & \multicolumn{7}{|c|}{ Average values of chronic stage at (35DPI) } \\
\hline & \multirow{2}{*}{ ELISA IgM/ IgG } & \multicolumn{6}{|c|}{ Tachyzoites load/10mg tissue } & \multirow[t]{2}{*}{ ELISA IgM/ IgG } & \multicolumn{6}{|c|}{ Bradyzoites load/10mg tissue } \\
\hline & & $\mathbf{L}$ & $\mathbf{K}$ & $\mathbf{P}$ & $\mathbf{U}$ & B & M & & $\mathbf{L}$ & $\mathbf{K}$ & $\mathbf{P}$ & $\mathbf{U}$ & B & M \\
\hline Type I & $\begin{array}{l}(0.553 \pm 0.36 \\
/ 0.290 \pm 0.20)\end{array}$ & 772 & 538 & 585 & 473 & 175 & 56 & $\begin{array}{l}(0.347 \pm \\
0.28 \\
/ 0.360 \pm 0.22)\end{array}$ & 19 & 63 & 19 & 96 & 430 & 152 \\
\hline Type II & $\begin{array}{l}(0.344 \pm 0.26 \\
/ 0.132 \pm 0.16)\end{array}$ & 214 & 277 & 164 & 143 & 21 & 11 & $\begin{array}{l}(0.280 \pm 0.19 \\
/ 0.312 \pm 0.24)\end{array}$ & 28 & 17 & 37 & 241 & 646 & 118 \\
\hline
\end{tabular}


Fadaly et al., Afr. J. Infect. Dis. (2015) 9(2): 32 - 38

http://dx.doi.org/10.4314/ajid.v9i2.3

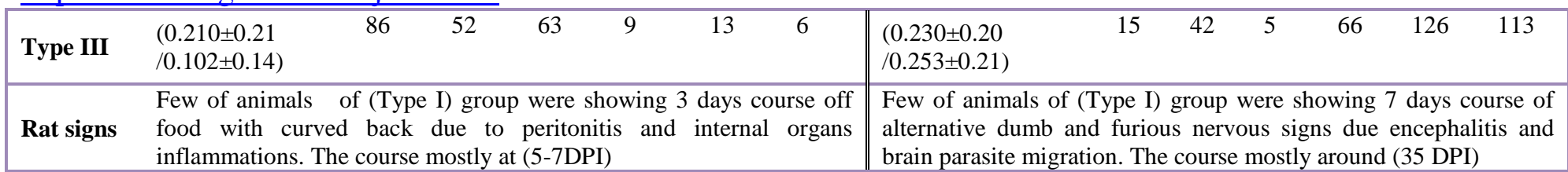
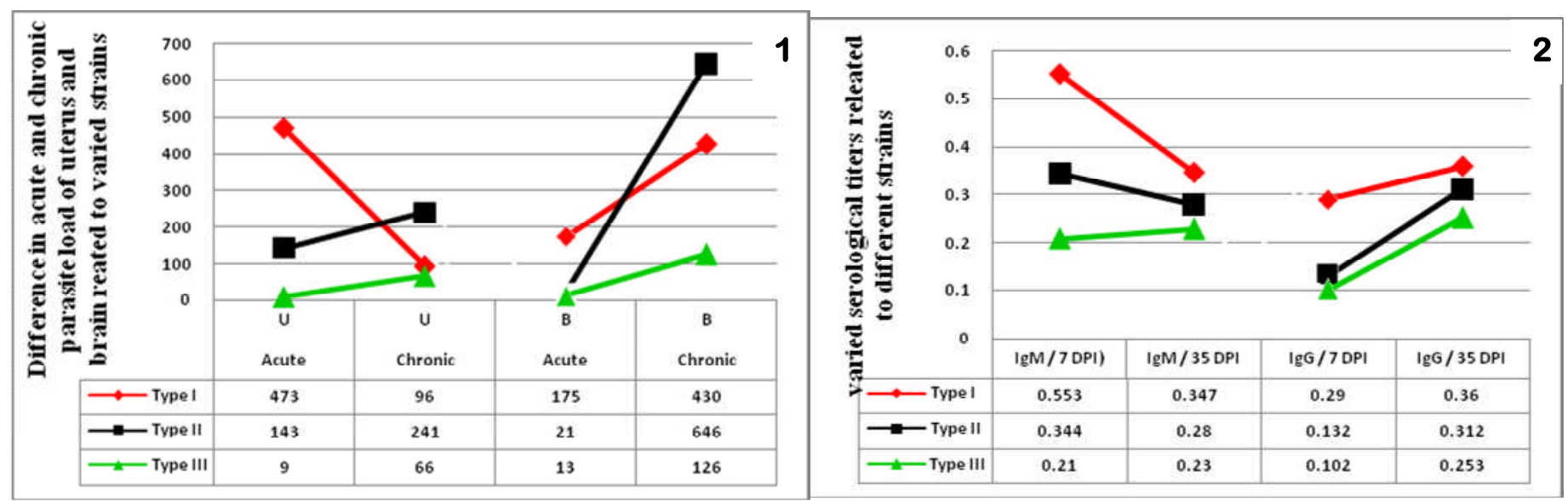

Figure $1 \& 2$ : Average parasite load and serological variations corresponding to different strain types.

Table 2 and Figures ( 1 and 2) showed an increase of the parasitic load especially in liver acute cases followed by pancreases and kidney $772,585 \& 538$ respectively while for type II kidney, liver and $277,214 \& 164$, and type III shows liver, pancreases and kidney 86 , 63\&52 respectively. In chronic cases, a high concern of parasitic load at brain tissues $430,646, \&, 126$, representing type I, type II and type III respectively were shown.

In the present study also appeared an elevation of $\operatorname{IgM}(0.553 \pm 0.36,0.344 \pm 0.26 \& 0.210 \pm 0.21$ titer recorded superior values than the analogous Ig G0 $.290 \pm 0.20,0.132 \pm 0.16 \& 0.102 \pm 0.14$ specially type I, signifies powerful success of tachyzoites stage re-conversion, able to exciting blood phase relapse, and reverts acute parasitemia, which was confirmed by the augmented titer of IgM. $347 \pm 0.28$ immunoglobulin. In contrast, latency was confirmed as corresponding to the significance higher IgG $360 \pm 0.22,312 \pm 0.24 \& 253 \pm 0.21$ representing type I, type II \& type III respectively than IgM $0.347 \pm 0.28$, $0.280 \pm 0.19 \& 0.230 \pm 0.20 \mathrm{re}$. Also the results ensured that strain types were validated as the major latent stimulus during chronic phase.

\section{Histo-pathological findings}

Liver

a). Microscopically, in group of rats treated with $T$. gondii Type I strain tachyzoites, the liver showed severe diffused degenerative changes mainly vacuolar type throughout the hepatic parenchyma (Fig.3) associated with focal parenchyma

b). In group of rats treated with $T$. gondii Type II strain bradyzoites, there were vacuolar degeneration of hepatic cells associated with mononuclear inflammatory cells infiltration of portal area (Fig.4), in addition to focal accumulations of mononuclear inflammatory cells mainly lymphocytes and macrophages associated with hepatic cords dissociation. The portal areas were greatly infiltrated with lymphocytes.

Kidney

Hyperplasia of biliary epithelium and proliferation of the bile duct were the results of the group-type III at all the organs. Group of rats treated with $T$. gondii Type I strain tachyzoites showed focal interstitial aggregations of mononuclear cells mainly lymphocytes (Fig.5). The renal tissue revealed severe focal necrosis and desquamation of the epithelial lining of the most renal tubules. There were dilatation and congestion of renal blood vessels and degeneration of glomerular tufts Mononuclear. Nearly same picture was seen in rats treated with T.gondii Type III strain tachyzoites.

Lungs

The lungs in most cases of both groups treated with RH strains and type I and III showed diffuse interstitial pneumonia (Fig.6). The alveolar wall revealed hyperplasia and metaplasia of the epithelial lining of the alveoli (Fig.6) associated with infiltration of mononuclear inflammatory cells in the alveolar septa with the accumulation of mononuclear cells and some leukocytes in the alveolar lumen.

In addition the lungs of female rats infected by $T$. gondii Type II strain bradyzoites showed severe congestion and dilatation of the pulmonary blood vessels as observed (Fig.7).

\section{Uterus}

In group of rats treated with T. gondii Types I and III, uterus showed focal desquamation of surface epithelium (Fig.8) associated with diffuse edema and reduction in the uterine glands number. Some of the uterine glands were atrophied and less branched with narrow lumen. Diffuse leukocytic infiltration of mononuclear inflammatory cells mainly lymphocytes and macrophages were seen in the endometrial stroma. Endometrium of female rats infected by T.gondii Type I strain tachyzoites, showing atrophy of uterine glands with periglandular mononuclear inflammatory cells infiltration associated with diffuse edema (Fig.9). 
The brain

Brain of female rats infected with $T$. gondii Type I strain tachyzoites, showed astrocytic edema associated with congestion of cerebral blood as observed (Fig.10). Focal glaiosis and the congestion of blood vessels were observed (Fig.11)

Brain of female rats treated with T. gondii Type III, showed perivascular edema associated with the congestion of cerebral blood vessels (Fig.12)

\section{The heart}

The heart muscles show degenerative changes with interstitial edema (Fig.13). The heart of female rats infected with $T$. gondii Type I and III strain tachyzoites showed clusters of tachyzoites between heart muscles (Fig.14, 15). On the other hand, the liver in groups treated with $T$. gondii Type II strain, showed mild focal vacuolar degeneration of hepatic cells. The lungs showed mild interstitial pneumonia and mild congestion of blood vessels. The kidneys were more or less within normal.

\section{Discussion}

Infection with $T$. gondii during pregnancy may lead to severe complications, if the infection is not fatal to the fetus (Remington et al., 1995). Therefore, emphasis is placed on preventive measures and early diagnosis for the prevention of severe complications of infection. Serologic screening in women during pregnancy is an important way to reducing maternal diffusion in the fetus. Seropositive women during pregnancy must not be exposed to treatment unless interpretation of $\mathrm{IgG}$ and $\mathrm{IgM}$ were done; treatment of at risk latent reactive cases or primary infected women with spiramycin to reduced congenital transmission (Barbosa et al., 2009). Early diagnosis in the mother, the fetus and the newborn is necessary to prevent human toxoplasmosis. The elevated IgG immune-competent women will not require treatment.

Serological examination was done to detect Toxoplasma-specific antibodies and to determine infection level with Toxoplasma and the Serologic testing is the routine method of diagnosis and results require a careful interpretation. So the Interpretation of $\operatorname{IgG}$ and $\operatorname{IgM}$ (Table - 2); IgM test essentially excludes recent infection, but a positive IgM test is difficult to interpret because Toxoplasma-specific IgM antibodies may be detected for as long as 18 months after acute acquired infection due to the reactivation of the dormant parasite (Remington et al., 2006).

In this study ELISA was used for sero-monitoring of varied IgM \& IgG immunoglobulin titers in the acute and chronic infected groups. Usually, IgM is begun hours after primary infection and earlier than IgG, followed by sequence decline parallel with progression elevated IgG titers, therefore the IgM titer normally not exceeding the matching IgG titer except at the starting of both primary or opportunistic infection (Louis et al., 2007). So, in the present study the sharp elevated IgM titer recorded superior values than the analogous IgG, signifies powerful success of tachyzoites stage re-conversion, able to exciting blood phase relapse ,and reverts acute parasitemia, which was confirmed by the augmented titer of IgM immunoglobulin. In contrast, latency was confirmed corresponding to the significance higher IgG than IgM. Also the results ensure that strain types validated as the major latent stimulus during chronic phase.

Histo-pathological examination of tissues of rats experimentally infected with T.gondii Type I (RH strain), revealed severe diffuse degenerative changes mainly vacuolar type, in addition to focal accumulations of mononuclear inflammatory cells mainly lymphocytes and macrophages, in addition to great infiltration with lymphocytes., causing encephalitis, endometritis, nephritis. With special emphasis to cerebral and uterine lesions, compatible mice signs with (RH-Type were observed); showing 3 days course off food with curved back due to peritonitis and internal organs inflammations, mostly at (57DPI). Also, (RH-Type I); showed 7 days course of alternative dumb and furious nervous may be due to due encephalitis and brain parasite migration, mostly around (35 DPI). (Carneiro et al., 2009).

Similar results were recorded by Barakat et al. (2001), Haziroglu et al. (2003), desouky et al. (2005), reported that the mentioned above toxoplasmic lesions were associated with presence of tissue cysts, and such pathological alters could be related to the penetration of the acute tachyzoites, which rapidly multiply by endodyogeny in nucleated host cells (Frenkel, 1988). There is no available evidence that T. gondii produces toxins (Topley and Wilson, 1998), where $T$. gondii tachyzoites actively have penetrated and rapidly multiplied in wide variety of host cells and eventually cause lysis which resulted in foci of necrosis (Dubey and Beattie, 1988). Frankel (1988) reported that tachyzoites were the principal pathogenic form of toxoplasma, they actively invaded host cells by means of an anterior organelle and released of proteolytic enzymes causing local disruption of host cell plasmalemma.

The desquamation of the endometrium epithelium and atrophy of uterine glands were seen in the uteri of infected rats, particularly with $\mathrm{RH}$ strain. Such uterine changes might be due to failure in ovarian activity. In this respect, Stahl et al. (1995) stated that chronically infected mice for three months with T. gondii developed ovarian and uterine atrophy and related this condition to pituitary gonadotrophin insufficiency resulting from the inhibition of gonadotrophin releasing hormones $(\mathrm{GnRH})$ from hypothalamus and they concluded that the inhibition of GnRH is due to cytokines released at periphery in response to when a parasite reached the hypothalamus.

\section{Pathological legends for Figures 3-15 below}

(Fig.3): Liver of female rats infected by T.gondii Type I strain tachyzoites, showing diffuse vacuolar degeneration of hepatic cells, associated with individual cell necrosis (H\&E stain, X 200).

(Fig.4): Liver of female rats infected by T.gondii Type II strain bradyzoites, showing vacuolar degeneration of hepatic cells associated with mononuclear inflammatory cells infiltration of portal area (H\&E stain, X 200).

(Fig.5): Kidney of female rats infected by T.gondii Type I strain tachyzoites, showing necro-biotic changes of lining epithelium of renal tubules. (H\&E stain, X200).

(Fig.6): Lung of female rats infected by T.gondii Type I strain tachyzoites, showing interstitial congestion associated with hyperplasia of alveolar wall (H\&E stain, X200).

(Fig.7): Lung of female rat infected by T.gondii Type III strain bradyzoites, showing hemorrhage and congestion of blood vessels (H\&E stain, X100).

(Fig.8): Uterus of female rat infected by T.gondii Type I strain tachyzoites, showing focal desquamation of surface epithelium associated with diffuse edema (H\&E stain, X 200).

(Fig.9): Uterus of female rat infected by T.gondii Type I strain tachyzoites, showing atrophy of uterine glands with periglandular mononuclear inflammatory cells infiltration associated with diffuse edema (H \&E stain, X200). 
http://dx.doi.org/10.4314/ajid.v9i2.3

(Fig.10): Brain of female rat infected by T.gondii Type I strain tachyzoites, showing astrocytic edema associated with congestion of cerebral blood vessels (H\&E stain, X200).

(Fig.11): Brain of female rat infected by T.gondii Type I strain tachyzoites, showing gloisis and congestion of blood vessels (H\&E stain, X100).

(Fig.12): Brain of female rat infected by T.gondii Type III strain, showing perivascular edema associated with congestion of cerebral blood vessels (H\&E stain, X100).

(Fig.13): Heart of female rat infected by T.gondii Type I strain, showing degeneration associated with interstitial edema between muscles fibers (H\&E stain, X 200).

(Fig.14): Heart of female rat infected by T.gondii Type I strain, showing cluster of tachyzoites between heart muscles. (H\&E stain, X 1000).

(Fig.15): Heart of female rat infected by T.gondii Type III strain, showing cluster of tachyzoites between heart muscles. (PAS stain, X1000).

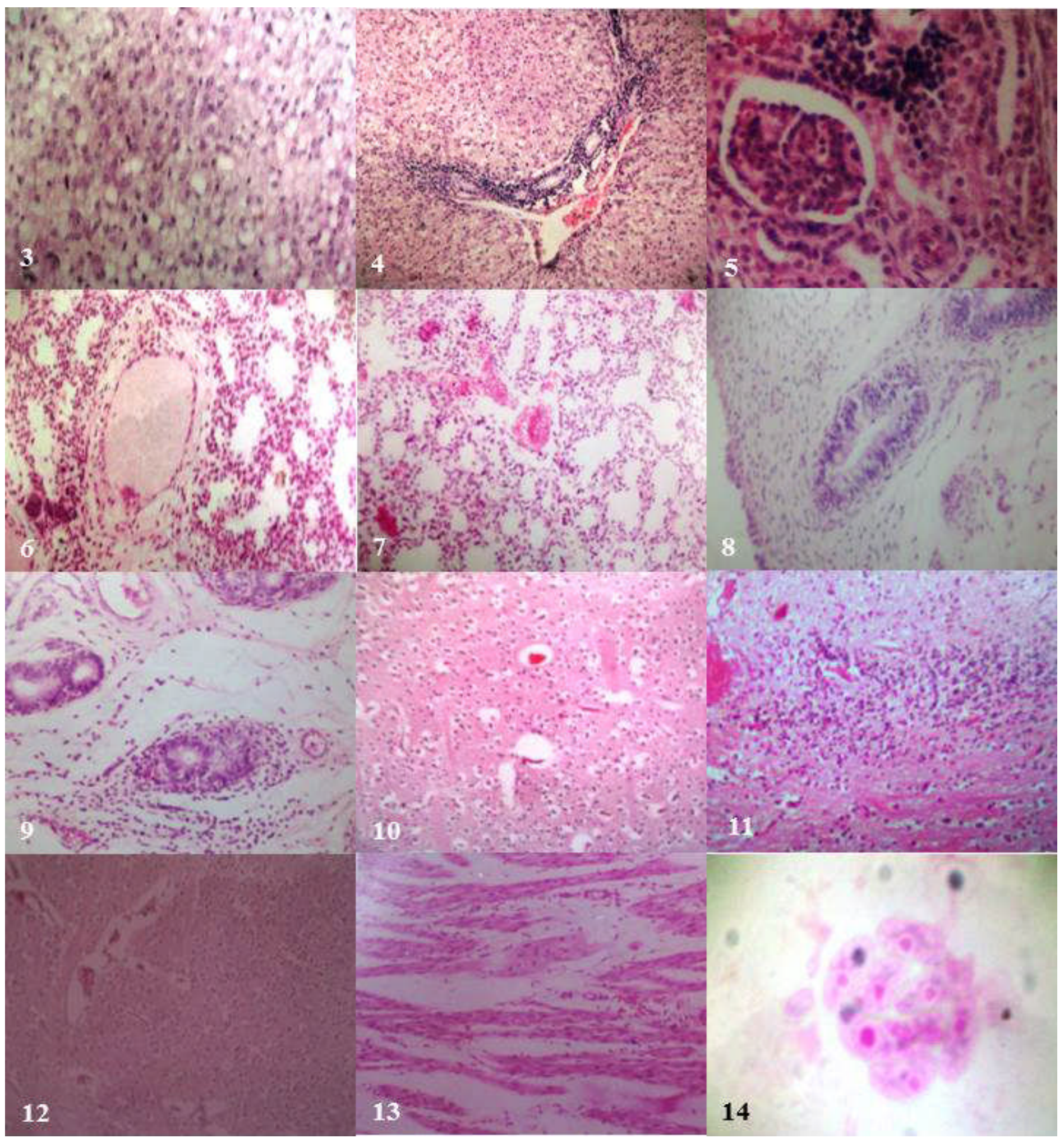




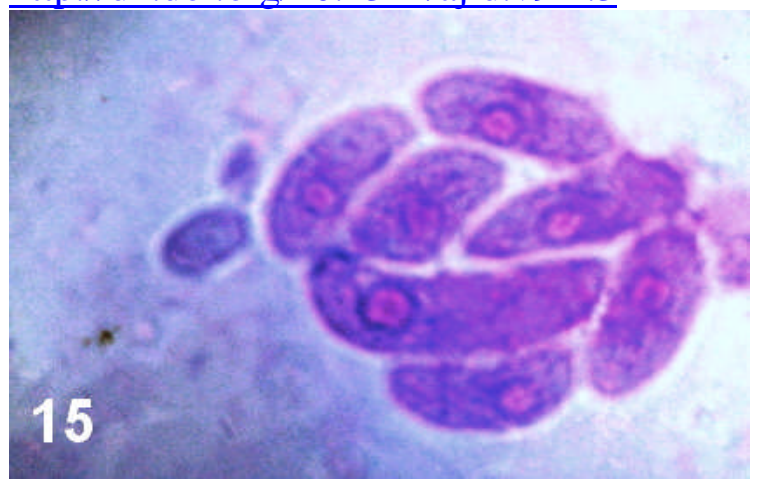

The results clarified the powerful activity of type I to transmuted forming tissue cyst followed by the higher ability of latency via bradyzoitestachyzoites re-conversion later on (Howe and Sibley, 1994). The higher ability of latency may be related to higher susceptibility of females due to deviation of sex steroid levels suspected to have stimulated silent bradyzoites to liberate from tissue cysts, and motivate the recurrent acute symptomatic stage, throw tachyzoites re-conversion, with subsequent increase in parasite load (Dubey \& Shen, 1991 and Craig et al., 2001), however, the result explained why toxoplasmosis was more prevalent in woman.

It is well known that female rats have higher Progesterone and Estradiol levels than male rats, thus alter immune system and induce higher susceptibility to parasite attacks. Whereas, sex steroids hormones worsens toxoplasmosis in females than male ones, and play a vital role during parasite infections, mainly through modulation of the host immune endocrine network (IEN) or direct regulation of parasite reproduction and differentiation (Cabrera-Muñoz et al. 2010). Also was confirmed as stimulating factor for promoting higher uterine and brain cysts (Cabrera-Muñozet al., 2010) plus the higher incidence of toxoplasmic encephalitis was recorded within females than in males, this support that female hormones possibly opportunist latent toxoplasmosis (Thapar et al., 1988), and was confirmed to stimulate higher parasite load in guinea pigs (Flori, 2002).

Although, type I strain group were reproduced in human foreskin fibroblasts but a third type strain faster than types II or III does (Kong et al., 2003), but analysis in France signify that cystogenic strains were validated as the more dominate human congenital types (Couvreur et al., 1976). The current study agree \& confirm that RH tachyzoites expressed a higher brain and uterine draw back than type II \& III during acute infection, represented in the higher percent of the parasite load, and denoted that the reconverted tachyzoites of cystogenic types are less serious to induce acute uterine or cerebral phase, and consequently to less incidence of encephalitis, or pregnant complications. Our results agreed with the higher persistence of tissue cysts in rat progeny (Dubey 1998). Also, results were corroborated that a woman congenital toxoplasmosis usually set synchronized with acute infection (Tenter et al. , 2000, Ajzenberg et al., 2002, Boothroyd and Grigg 2002). Though, there is no guarantee that the dramatic variation among virulent types in rat, might extend to humans.

It could be concluded that; T. gondii cerebral and uterine infestation within rats were synchronized with higher progression of IgM than IgG. Also, it signifies acute type I tachyzoites as higher pronounced cerebral and uterine pathological lesions than chronic cystogenic types II.

\section{References}

1. Abd El-Razik, K.A., AL-Humiany, A.A., Ahmed, W.M. Barakat, A.M.A. and ELfadaly H.A. (2011): Investigations on non Brucella Abortifacients in Small Ruminants in Saudi Arabia with Emphasis on Zoonotic Causes. Global Veterinaria 6 (1): 25-32.

2. Ajzenberg, D., Anne-Laure Bañulsb., Tibayrencb, M. and Marie Laure Dardéa. (2002). Microsatellite analysis of Toxoplasma gondii population shows a high polymorphism structured into two main clonal groups. Internal Journal of Parasitology 32:7-38.

3. Bancroft, J.D., Stevens, A. and Turner, D.R. (1996). Theory and Practice of Histological Techniques. 4th ed., Churchill Livingstone, New York, Edinburgh, London, Melbourne, San Francisco, Tokyo.

4. Barakat, A.M., Desouky, H.M., Abd El-Aziz, M.M. and Abd El-Aal, A.A. (2001). "Some serological, hormonal and pathological studies on experimental Toxoplasmosis in male rabbits." J. Egypt. Vet. Med. Ass. 61:165-182.

5. $\quad$ Barakat, A. M., Abdel-Aal, A., Eid, R.A.A. and Abd El-Razik, K.A. (2005). "Effects of Toxoplasma gondii on reproduction in rabbits." J .Egypt. Vet. Med, Assoc.65, no 3:39-55.

6. Barbosaa, IR. de-Carvalho, C.M. Holandab, X. and de-Andrade-Neto, VF. (2009). Toxoplasmosis screening and risk factors amongst pregnant females in Natal, northeastern Brazil. Trans Roy Soci Trop Med Hyg 103, 377-382.

7. Boothroyd, J.C. (2009). Toxoplasma gondii: 25 years and 25 major advances for the field. Int. J. Parasitol. 39 ( 8): $935-946$.

8. Boothroyd, J.C. and Grigg, M.E. (2002). Population biology of Toxoplasma gondii and its relevance to human infection: do different strains cause different disease? Current Opinion Microbiology 5: 438-442.

9. Cabrera-Muñoz, E., Escobedo, G., Guzmán, C. and Camacho-Arroyo, I. (2010). Role of Progesterone in HIV and Parasitic Infections. The Open Neuroendocrinology Journal3: 137-142.

10. Carneiro , A. C .A .V., Carneiro, A.M.G. Gouveia Guimarães, A.S., Marques, A.P.R., Vilas-Boas, L.S. and Vito, R.W.A. (2009). Seroprevalence and risk factors of caprine toxoplasmosis in Minas Gerais, Brazil . Vet. Parasitol. 160: $225-229$.

11. Craig, W., Walker, W. and Alexander, J. (2001). Sex-Associated Hormones and Immunity to Protozoan Parasites. American Society for Microbiology.

12. Couvreur, M.D. J., Desmonts, M.D.G. and Girre, M.D.G.Y. (1976). Congenital toxoplasmosis in twins: A series of 14 pairs of twins: Absence of infection in one twin in two pairs. The Journal of Pediatrics 80 (2):235-240.

13. Desouky, H.M., Barakat, A.M. and Abd El-Razik, K.A. (2005). "Toxoplasma gondii in rabbit-does: serological, hormonal, pathological and molecular aspect" Egypt. J. Comp. Path. \& Clinic. Path. 18:145-167.

14. Dubey, J.P. (1998). Comparative infectivity of Toxoplasma gondii bradyzoites in rats and mice. Journal of Parasitology 84:1279-1282.

15. Dubey, J.P. and Shen, S.K. (1991). Rat model of congenital toxoplasmosis. Infection \& Immunity 59: 3301-3302 .

16. Dubey, J.P. and Beattie, C.P. (1988). "Toxoplasmosis of animals and man.” CRC press, Bocaraton, Florida, USA. 
http://dx.doi.org/10.4314/ajid.v9i2.3

17. Drury RAB, Wallington EA (1980). Carleton's histological technique. Oxford University Press, Oxford New York Toronto, pp. 140-142. pp 496497

18. Drury et al., (1976). R.A. Drury, E.A. Wallington, R. Cancerson ; Carlton's Histopathological Techniques (fourth ed) Oxford University Press, Oxford. London, New York (1976).

19. Elfadaly, H.A. (2007). Advanced epidemiological studies on toxoplasmosis in animals and man. PhD.V.Sc, Thesis, Zoonotic Diseases Department,Cairo University.

20. Elfadaly, H.A., Hassanain, M.A., Shaapan, R.M., Ashraf, M.and BarakatToaleb, N.I. (2012). Serological and Hormonal Assays of Murine Matern Fetal Toxoplasma gondii Infection with Emphasis on Virulent Strains; World Journal of Medical Sciences 7 (4): 248-254.

21. Flori, P. (2002). Experimental model of congenital toxoplasmosis in guinea-pigs: use of quantitative and qualitative PCR for the study of maternofetal transmission. Journal of Medical Microbiology 51: 871-878.

22. $\quad$ Frenkel, J.K. (1988). "Pathology-siology of toxoplasmosis.” Parasit. Today 4:273-278.

23. Godard, I., Darcy, F., Deslee, D., Dessaint, J.P. and Capron, A. (1990). Isotypic profiles of antibody Responses to Toxoplasma gondii infection in rats and mice: kinetic study and characterization of targetantigens of immunoglobulin A antibodies. Infection \& Immunity 58: 2446-2451.

24. Hassanain, M .A., Elfadaly, H.A., Shaapan, R.M., Hassanain, N.A. and Barakat, A.M.(2012): Biological Assay of Toxoplasma gondii Egyptian Mutton Isolates. International Journal of Zoological Research 7 (4): 330-337.

25. Hassanain, M .A. , Elfadaly, H.A., Hassanain, N.A., Shaapan, R.M., Barakat, A.M. and Abd El-Razik, K. A. (2012): Serological and Molecular Diagnosis of Toxoplasmosis in Human and Animals. World Journal of Medical Sciences 9 (4): 243-247.

26. Haziroglu, R.,Altintas, K.,Atasever, A.,Glulbahar, M.Y. and Recai Tunca, O.K. (2003). "Pathological and immunological studies in rabbits experimentally infected with Toxoplasma gondii.” $\quad$ Turk. J. Anim. Sci. 27:285-293.

27. Howe, D.K. and Sibley, L.D.( 1994). Toxoplasma gondii: analysis of different laboratory stocks of the RH strain reveals genetic heterogeneity. Experimental Parasitology 78:242-245.

28. Jones, J.L., Lope, Z.A., Wilson, M., Schulkin, J. and Gibbs, R. (2001). Congenital toxoplasmosis: a review. Obstet. Gynecol. Surv. 56, $296-305$.

29. Kong, J.T., Grigg, M. E., Lyle, Uyetake, Parmley, S. and Boothroyd, J.C. (2003). Serotyping of Toxoplasma gondii infections in humans using synthetic peptides. Journal of Infectious Diseases 187: 1484-1495.

30. Kovari, H., Ebnöther, C., Schweiger A, Berther N, Kuster H, Günthard HF. (2010). Pulmonary toxoplasmosis, a rare but severe manifestation of a common opportunistic infection in late HIV presenters: report of two cases. Infections Post Transplant.; 38(2):141-4.

31. Lind, P., Haugegaard, J, Wingestrand A. and Henriksen, S.A. (1997). The time course of the specific antibody response by various ELISAs in pigs experimentally infected with Toxoplasma gondii . Vet Parasitol. 71: 1-5.

32. Louis M. Weiss and Kami Kim (2007). Toxoplasma gondii; the Model Apicomplexan: Perspectives and Methods; Copyright Elsevier Ltd. ISBN: 978-0-12-369542-0.

33. Remington, J.S., Mcleod, R., Desmonts, G., (1995). Toxoplasmosis. In: Remington JS, Klein JO (eds) Infectious Diseases of the Fetus and Newborn Infant. W. B. Saunders Co. Philadelphi, pp $140-267$.

34. Remington, J.S., McLeod, R., Thulliez, P. and Desmonts, G. (2006). Toxoplasmosis. Infectious Diseases of the Fetus and Newborn Infant. 5th ed. Philadelphia, PA: The WB Saunders Co.; p. 205-346.

35. Shaapan, R.M., Amal M. Abo-ElMaaty, Abd El-Razik, K.A. and Abd El-Hafez, S.M. (2012): PCR and Serological Assays for Detection of Toxoplasma Gondii Infection in Sport Horses in Cairo, Egypt. Asian journal of animal \& Veterinary Advances, 7(2):158-165.

36. Tenter, A.M, Heckeroth, A.R and Weiss, L.M. (2000). Toxoplasma gondii: from animals to humans. Int J Parasitol. Nov; 30(12-13):1217-1258.

37. Thapar, M., Kumari, GL.,Shrivastav, T.G. and Pandey, P.K. (1988). Hormonal control of implantation in guinea pigs. Steroids 52: 85-108.

38. Topley and Wilson (1998). "Microbiology and microbial infections" 9th Ed. Vol.5 (Parasitology) Eds: Francis E.G. Cox,Julius P. kreir and Derek Wakelin. Arnold Oxford University. Press, New York.

39. Zhou, X W., Blackman, M.J., Howell, S.A. and Carruthers, V.B. (2004). Proteomic analysis of cleavage events reveals a dynamic two-step mechanism for proteolysis of a key parasite adhesive complex. Mol. Cell Proteomics 3: 565-576. 